\title{
Blood filled cyst of the papillary muscle of the mitral valve producing severe left ventricular outflow tract obstruction
}

\author{
Regional \\ Cardiothoracic Unit, \\ Groby Road Hospital, \\ Leicester \\ I R Arnold \\ P J B Hubner \\ R K Firmin \\ Correspondence to \\ Dr Ian R Arnold, \\ Regional Cardiothoracic \\ Unit, Groby Road Hospital, \\ Groby Road, Leicester \\ LE3 9QE.
}

\author{
Ian R Arnold, Peter J B Hubner, Richard K Firmin
}

\begin{abstract}
A 46 year old man was found to have left ventricular outflow obstruction caused by a blood-filled cyst attached to the anterior papillary muscle of the mitral valve. It was successfully excised.
\end{abstract}

Small blood filled cysts of cardiac valves are commonly found in infants at necropsy, but are rare after the first year of life. They are believed to be cystic dilatations at the end of canals lined with endothelium in the substance of the valves. ${ }^{12}$ We report a cyst of the mitral valve presenting as left ventricular outflow obstruction in an adult; it was successfully resected.

\section{Case report}

A 46 year old farmer gave a 5 year history of chest tightness and mild faintness on heavy exertion such as playing hockey or lifting bales of hay. There was no history of syncope. He had not had rheumatic fever.

On examination he was of lean build, apyrexial, and there was no finger clubbing. The pulse was regular but jerky. Blood pressure was $100 /$ $60 \mathrm{~mm} \mathrm{Hg}$. There was a systolic thrill palpable over the left sternal edge. There was a loud, harsh ejection systolic murmur heard throughout the precordium but greatest at the left sternal edge. It did not radiate to the carotid arteries.

The electrocardiogram showed sinus rhythm, an axis of $+45^{\circ}$, left ventricular hypertrophy ( $\mathrm{S}$ wave in $\mathrm{V} 2$ and $\mathrm{R}$ wave in $\mathrm{V} 5=6 \mathrm{mV}$ with no $T$ wave changes), and no left atrial hypertrophy. The chest $x$ ray showed a cardiothoracic ratio of $50 \%$, but this had increased from $44 \%$ five years earlier. There was no prominence of the ascending aorta or left atrium. The haemoglobin concentration was $152 \mathrm{~g} / \mathrm{l}$, the erythrocyte sedimentation rate was $2 \mathrm{~mm} / \mathrm{h}$, and serum protein electrophoresis was normal.

Echocardiography showed an aortic valve that was not thickened or disorganised but showed prominent early systolic closure (fig 1a). There was a non-rheumatic mitral valve with systolic anterior motion of the anterior leaflet (fig lb). There was mild concentric left ventricular hypertrophy. Cross sectional echocardiography showed a spherical mass $(2 \cdot 4$ $\mathrm{cm}$ in diameter) with an echolucent centre within the left ventricular cavity (fig 2 ). It seemed to be attached to the anterior leaflet of the mitral valve. In systole it prolapsed into the left ventricular outflow tract. The cyst wall could be seen on the $M$ mode echocardiogram as a fine echo abutting the septum (fig $1 \mathrm{~b}$ ). Doppler studies showed a turbulent high velocity systolic jet within the left ventricular outflow tract and no mitral regurgitation was detected.

At cardiac catheterisation the pulmonary capillary wedge pressure was $24 / 8 \mathrm{~mm} \mathrm{Hg}$, mean 16 . The pulmonary artery pressure was $44 / 24 \mathrm{~mm} \mathrm{Hg}$, mean 24 . There was no mitral valve gradient. There was a gradient of $140 \mathrm{~mm} \mathrm{Hg}$ across the left ventricular outflow tract, falling to $110 \mathrm{~mm} \mathrm{Hg}$ after $0.3 \mathrm{mg}$ of phenylephrine was given into the pulmonary artery. The withdrawal gradient across the left ventricular outflow tract was $140 \mathrm{~mm} \mathrm{Hg}$ (left ventricle body $240 / 20$, left ventricle outflow tract $100 / 20$, and aorta $100 / 65$ ). Left ventricular cineangiography showed a mobile spinerical mass within the left ventricle moving

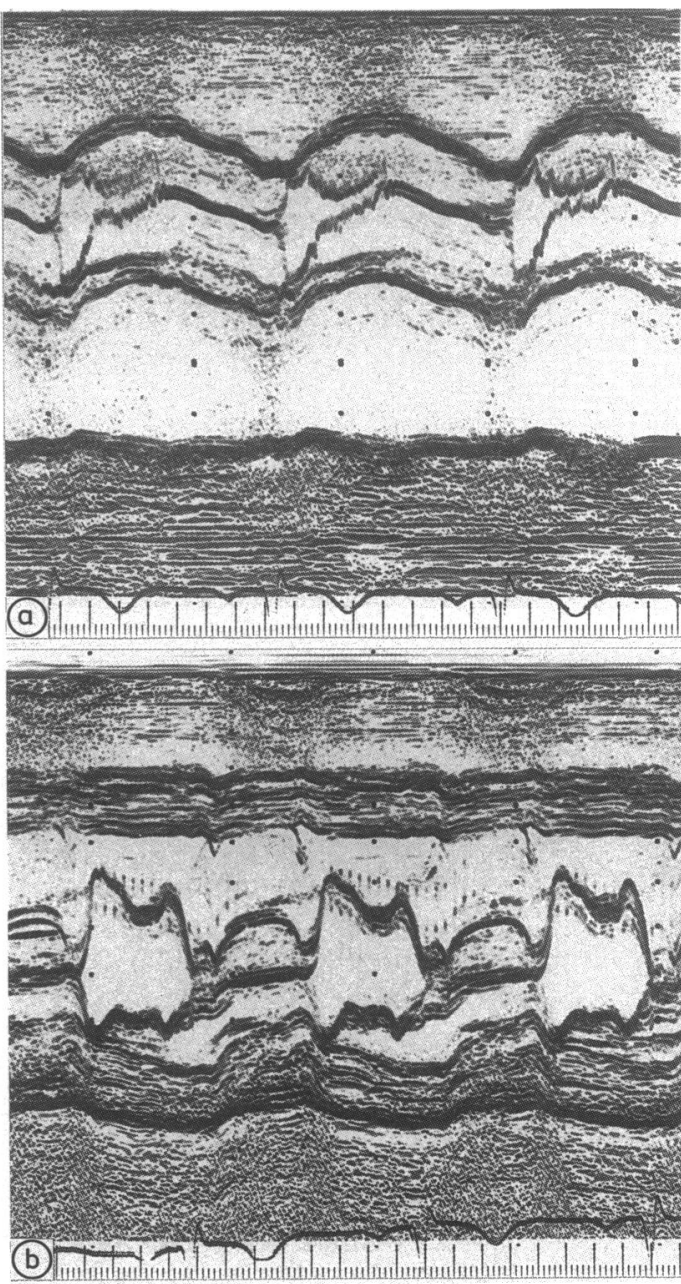

Figure $1 M$ mode echocardiograms showing parasternal views through (a) aortic root and (b) mitral valve. 
Figure 2 Cross sectional echocardiogram showing apical view. S, septum; $L V$, left ventricle; $C Y$, cyst.

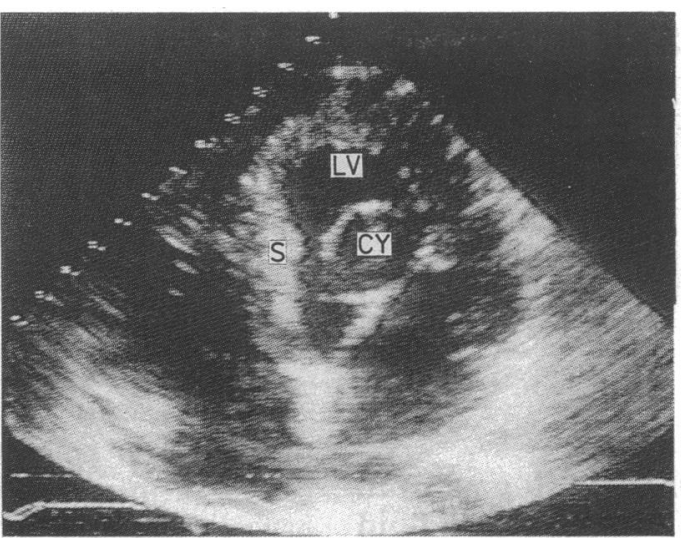

into the left ventricular outflow tract in systole. It seemed to be attached to the anterior leaflet of the mitral valve.

At operation, through an aortotomy, a smooth oval bluish mass ( 3.2 by $2.5 \mathrm{~cm}$ across), was found attached by a stalk to the tip of the anterior papillary muscle of the mitral valve. It was initially thought to be a tumour and to ensure complete clearance, the papillary muscle and anterior leaflet of the valve were excised through a standard left atrial approach and a $29 \mathrm{~mm}$ St Jude prosthesis was inserted.

The patient made an uneventful postoperative recovery and at follow up five months later was symptom free and working full time.

Histological examination showed a blood filled cyst, the wall of which was lined by an attenuated endothelium but with no evidence of epithelium. The wall itself was composed of fibrous tissue with no smooth muscle or organised elements within it.

\section{Discussion}

Leatherman et al reported the anatomical and pathological features of a blood cyst attached to the anterior leaflet of the mitral valve in a 39 year old woman that was successfully excised without requiring mitral valve replacement. ${ }^{3}$ No outflow tract gradient was shown in their case.

Hauser et al reported a blood filled cyst attached to the tip of the anterolateral papillary muscle of the mitral valve in a 27 year old man that was successfully excised with the mitral valve. ${ }^{4}$ The diagnosis was made on cross sectional echocardiography, and catheterisation was not performed. Liese et al, Cumming and Ferguson, and Jimenez-Martinez et al reported blood filled cysts attached to the pulmonary valve diagnosed at angiography and successfully excised at operation..$^{5-7}$

Our patient presented with symptoms and signs suggestive of either severe mitral regurgitation or left ventricular outflow tract obstruction. Early closure of the aortic valve and systolic anterior movement of the mitral valve on $M$ mode echocardiography suggested hypertrophic obstructive cardiomyopathy but the left ventricular hypertrophy was only mild and symmetrical. Cross sectional echocardiography showed that a most unusual spherical mass with echolucent centre attached to the mitral valve was the cause of the left ventricular outflow obstruction. The obstruction was confirmed by the demonstration of a $140 \mathrm{~mm} \mathrm{Hg}$ gradient across the left ventricular outflow tract at catheterisation. The systolic anterior motion of the mitral valve may have been caused by a direct mechanical effect of the cyst pulling at its attachment rather than by the Venturi effect that is suggested to explain such motion in hypertrophic obstructive cardiomyopathy.

At operation the papillary muscle was excised to ensure adequate clearance of what was initially thought to be a vascular tumour. We suggest that the diagnosis of a blood-filled cyst should be considered if echocardiography shows a spherical mass with an echolucent centre; it may prove possible to remove it without the need to replace the mitral valve.

To our knowledge this is the first report of a simple blood cyst causing confirmed left ventricular outflow obstruction, and the second description of the echocardiographic features of such a cyst.

We thank Dr A Fletcher for his assistance with the histopathology.

1 Mills SD. The occurrence of blood-filled cysts on the cardiac valves in infancy. $J$ Pediatr 1935;6:51-6.

2 Begg JG. Blood-filled cysts in the cardiac valve cusps in foetal life and infancy. J Pathol Bacteriol 1964;87:177-9. Leatherman L, Leachman RD, Hallman GL, Cooley DA Cyst of the mitral valve. Am J Cardiol 1968;21:428-30.

4 Hauser AM, Rathod K, McGill J, Rosenberg BF, Gordon S, Timmis GC. Blood cyst of the papillary muscle. $A m$ Cardiol 1983;51:612-3.

5 Liese GJ, Brainard SC, Goto U. Giant blood cyst of the pulmonary valve. N Engl J Med 1963;269:465-7.

6 Cumming GR, Ferguson CC. An elusive tumour of the pulmonary valve associated with a coronary arteriovenous fistula. J Thorac Cardiovasc Surg 1965;50:715-20.

7 Jimenez-Martinez M, Franco-Vazquez S, AnguloJimenez-Martinez M, Franco-Vazquez S, Angulo-
Hernandez O, Reta-Villalobos A. Blood cysts of the pulmonary valve producing pulmonic stenosis. $J$ Thorac Cardiovasc Surg 1968;56:165-9. 